\title{
Multiple Core Fibers versus Multiple Fibers Enabled Space Division Multiplexing Based Elastic Optical Networks: A Performance Comparison
}

\author{
Sridhar Iyer \\ Department of ECE, Jain College of Engineering, T.S. Nagar, Machhe, Belagavi, KA, India - 590014. \\ E-mail: sridhariyer1983@gmail.com
}

Received: 01 July 2019; Accepted: 22 July 2019; Published: 08 August 2019

\begin{abstract}
Space Division Multiplexing (SDM) based Elastic Optical Networks (EONs) (SDM-b-EONs) have been envisioned as a solution to both, the required upgradation of the single-mode fiber's network capacity which is constrained by the non-linear Shannon's limit and the capacity provisioning which will be necessitated by future diverse Internet traffic. The current SDM-bEON designs are based on the use of Multiple Fibers (MF) technology; however, recently the Multiple Core Fiber (MCF) technology has gained momentum after laboratory experiments conducted on the MCF models established much lower inter-core crosstalk values. In the current work, we focus on the design of a MCF enabled SDM-bEON for which, we formulate an Integer Linear Programming (ILP) model and then propose a heuristic algorithm to obtain the solutions for large sized networks in reasonable execution times. We then proceed to the performance comparison of the MCF and the MF enabled SDM-b-EON under the consideration of realistic parameters and network topologies which are characterized by different node(s) numbers and link distances. The obtained results demonstrate that the performance of the MCF enabled SDM-b-EON is very close to that of a MF enabled SDM-b-EON which implies that the network operators can benefit by utilizing the existing components for the MCFs rather than incurring any extra expense to provision the same traffic amount.
\end{abstract}

Index Terms -Elastic optical networks, space division multiplexing, inter-core crosstalk, ILP, heuristic.

\section{INTRODUCTION}

The ever growing diverse traffic and its related bandwidth requests have rendered the optical networks (OTNs) limited by the capacity. To quench the demands for large capacity and heterogeneous granularity by the traffic of the next-generation OTNs, the flexi-grid elastic optical networks (EONs) have been thoroughly investigated $[1,2]$. However, owing to its use of only two multiplexing dimensions, in the near future, the EONs will also lead to a crunch in the fiber capacity. The aforementioned scenario can be ameliorated by the adoption of space division multiplexing (SDM) which introduces a new dimension of 'space' and in which, many fibers are utilized in parallel thus provisioning an increase in the spectral resources which can be utilized [3]. The SDM based EONs (SDM-b-EONs) will hence be able to efficiently serve the next generation's applications and the Internet which have been anticipated to handle the traffic growth at a rate much greater than Petabit per second level [4] owing to their large bandwidth capacity and efficient accommodation of both, the low bit-rate (BR) lightpaths and the high BR super-channels [5].

Currently, the SDM-b-EON solutions proposed by both, the research community and the network operators is based on the extension of the already deployed networks which have been converted into infrastructures that are enabled by multiple fibers (MFs) wherein, each fiber link bundles many single mode fibers (SMFs) [6]. However, for the SDM-b-EONs to appeal economically, new fiber design solutions are required to ensure parallelization, which is a mandate in the SDM-b-EONs [7]. Amongst the many other existing solutions, SDM-b-EONs enabled by multiple core fibers (MCFs) are promising since such a technology (i) can provide higher spectrum usage flexibility in conjunction with the bandwidth scalability far beyond the non-linear Shannon's limit, and (ii) makes it possible to benefit from system components which are integrated, cost-effective, and already existing for the MCFs [7, 8]. Also, as already demonstrated in [5], owing to low levels of XT, the MCFs are completely flexible and hence there occurs a possibility of resorting to the use of both, spectral and spatial super-channel techniques. However, use of the MCF technology also implies (i) insertion of several single mode cores within a single fiber cladding, (ii) constraint of increase in core(s) number(s) essentially due to space non-availability within fiber cladding, and (iii) counteracting of degradations due to coupling among the cores i.e., inter-core crosstalk (ICXT), which can degrade the optical signal(s)' transmission reach (TR) to such an extent that it may be required to adopt a less efficient however, more robust 
modulation format (MF).

Many existing studies have addressed the design of EONs under the assumption of single core fibers, a survey of which is presented in [2]. However, with SDM introducing 'space' as a new freedom degree, the SDM-bEON scenario is very recent and hence, only few works exist on the design of SDM-b-EONs. The authors in [9] have experimentally evaluated a four node programmable multiple granularity SDM switching network using 'two' seven core MCFs. The obtained results demonstrate an adequate end to end performance on all the channels. In [10], the authors have proposed an Integer Linear Programming (ILP) based optimal method followed by the proposal of heuristic schemes for the routing, MF, core and spectrum assignment (RMFCSA) problem in a SDM-b-EON with an aim to reduce the maximum spectrum slices amount required in the MCF's any core. The results, which are obtained under the assumption of a 'three' core MCF considering estimations of ICXT, demonstrate a good approximation of the proposed heuristic with the optimal solution obtained from the formulated ILP model. The authors in [11] have proposed heuristic schemes for the similar problem as in [10] considering the optical white box and the black box devices. The aim of the proposed schemes is to jointly optimize the switching and the spectrum resource efficiency during provisioning of the demands requiring diverse capacity(s). The results are obtained considering a 'six' core MCF with the same ICXT estimations as in [10] and it is demonstrated that, the ICXT aware schemes improve the provisioned traffic volume significantly for the SDM network based on the technology of architecture on demand which is a scalable and cost-efficient solution for future SDM networks. In [3], the authors have evaluated the benefits of using SDM for dynamic bandwidth allocation in an EON. The main aims of the study are to (i) compare the spectral and spatial super channel assignment policies in a SDM network which relies on SMFs bundles, (ii) investigate the impact of MF choice on the performance, and (iii) study the performance of various SDM switching options. The results of the study show that (i) under the consideration of a multiple channel single MF system with $50 \mathrm{GHz}$ sub channel spectrum occupation, for both, spectral and spatial super channel assignment policies, the DP-8-QAM MF offers the best compromise between spectral efficiency (SE) and TR, (ii) with the consideration of a multiple channel MF system, network performance improvement can be obtained, and (iii) joint switching is able to offer similar performance as that provided by independent switching for particular network load profiles, while allowing a significant reduction in the number of wavelength selective switches. The authors in [7] have proposed a cost-effective Reconfigurable Optical Add/Drop Multiplexer (ROADM) architecture for SDMb-EONs which are enabled by the MCFs and also minimize the technological requirements and associated costs in exchange for demanding core continuity along the end to end communication. The authors have also proposed a heuristic algorithm for solving the RMFCSA problem which is compared to its ILP counterpart. The obtained results demonstrate that, in addition to decrease in the network expenditures, in terms of the maximum throughput, when the proposed ROADM architecture is deployed, approximately similar performance is obtained compared to when the existing ROADM architectures are operational in the network.

In the current work, we focus on the design of a MCF enabled SDM-b-EON. Owing to the adoption of a flexigrid technology, during heterogeneous BR(s) lightpath(s) assignment, the optimization problem aims to maximally use the spectrum of every core. Further, we consider an EON links system detailed in [5] which comprises of single core fibers amount that equals the cores amount within the MCFs. The aforementioned implies that in our current work (i) spatial super channels are not considered, and (ii) at every node, there occurs spatial demultiplexing of the incoming MCFs. As a key contribution which distinguishes our current work from the existing studies, we compare the performance of a MCF enabled SDM-bEON with that of a MF enabled SDM-b-EON under the consideration of realistic parameters and network topologies which are characterized by different node(s) numbers and link distances. To the best of our knowledge, there is no existing study in literature which has focused on such a comparison. We also advocate for our current work, since it is the first study which demonstrates that the performance of MCF enabled SDM-b-EON is close to that of a MF enabled SDM-b-EON and hence, shows that the network operators can indeed benefit by using the existing components for the MCFs rather than incurring any extra expense to provision the same traffic amount.

Rest of the paper is structured as follows: In Section II, initially, we detail the SDM-b-EON design using an ILP formulation followed by proposal of a heuristic algorithm in view of obtaining the solutions for large sized network. In Section III, we detail the SDM-b-EON scenario and various simulation assumptions followed by the discussion on various obtained simulation results. Finally, Section IV concludes the study.

\section{Design OF Space Division MULTIPLEXING BASED ELASTIC OPTICAL NETWORK}

In this section we detail the problem statement of the SDM-b-EON design. Initially, an ILP model is formulated to obtain the optimal solutions followed by the proposal of a heuristic algorithm to obtain the solutions for large sized network topologies in reasonable execution times.

\section{A. ILP Formulation}

To formulate the ILP model for the SDM-b-EON design, we define the following:

- A graph $G\left(N_{F-O X C}, E\right)$ which comprises of $N_{F-O X C}$ flexible Optical Cross Connects (FOXCs) set and $E$ unidirectional MCFs set that connects the neighboring nodes. In our study, we 
also assume that the F-OXCs have the capability of ensuring spectral continuity constraint i.e., the F-OXCs can perform any input core's spectral portion switching to any output core.

- At the network nodes, the deployed flexible Transponders (F-TPs) are assumed to operate at a
BRs set $B R$, and further, the F-TPs can use a MFs set $M F$ at any BR $b r \in B R$.

- The MCFs are assumed to consist of $\varepsilon$ cores. Further, an ordered Frequency Slots (FSs) set

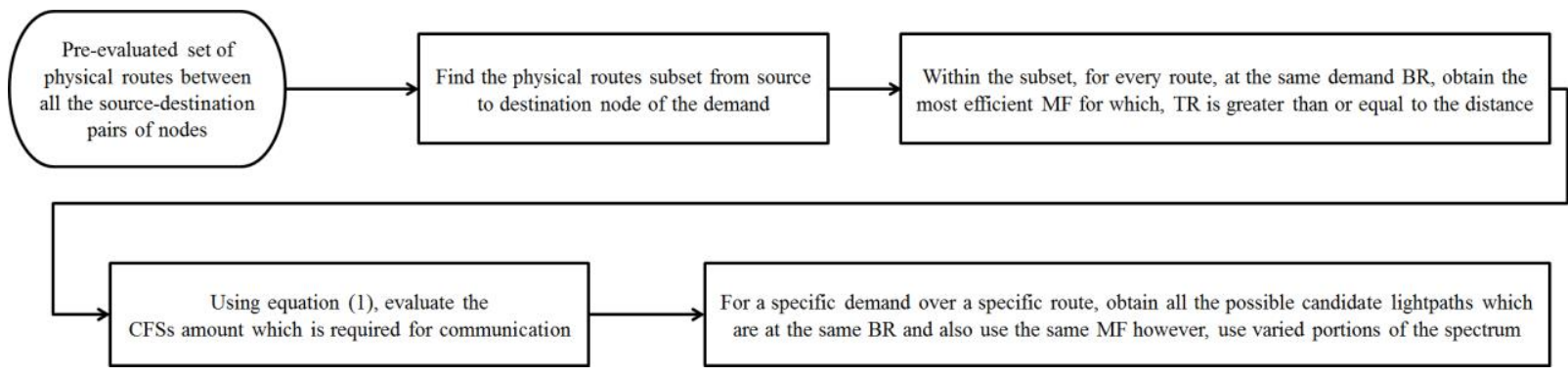

Fig.1. Steps to pre-evaluate the candidate lightpaths set for any demand.

$F S=\left\{f s_{1}, f s_{2}, \ldots \ldots, f s_{|F S|}\right\}$ is assumed to denote,

in a discrete format, the $\varepsilon$ cores' spectrum which is available in the network.

- The routes between all the source destination pairs of nodes comprised as a set $R$ is pre-evaluated. The distance and the $r \in R$ route's hops amount is denoted as $p d_{r}$ and $h o p_{r}$, respectively. Further, the routes' subset which comprises of MCF $e \in \varepsilon$ is denoted as $R_{e}$.

- The SDM-b-EON is assumed to have to provision a unidirectional demands set $D$ of arbitrary BR $b r \in B R$. Further, the permissible candidate lightpaths set which can provision a demand $d \in D$ is denoted as $C L_{d}$. For any demand $d \in D$, the corresponding $C L_{d}$ is preevaluated by using the steps of the flow chart shown in Fig. 1.

- Amongst the candidate lightpaths in $C L_{d}$, any candidate lightpath operating at a specific BR $b r \in B R$ and using a MF $m f \in M F$ is equivalent to a contiguous FSs (CFSs) set along a route $r \in R$ which assures that adequate amount of spectrum can be assigned to the candidate lightpath. The aforementioned CFSs amount is evaluated as follows:

$$
C F S s=\left(\frac{b r}{e f f_{m f}}+G B\right) / W
$$

where $e f f_{m f}$ is MF $m f^{\prime} s$ efficiency (in units of bits/s/Hz), $G B$ the spectrum guard bands needed between contiguous lightpaths (in units of $\mathrm{GHz}$ ), and $W$ the spectral width of FS (in units of $\mathrm{GHz}$ ).

- For a demand $d \in D$ that spans over the MCF $e \in \varepsilon$, the corresponding candidate lightpaths set is denoted as $C L_{d}^{e} \subseteq C L_{d}$. Further, for a demand $d \in D$ that uses FSs $f s \in F S$, the corresponding candidate lightpaths set is denoted as $C L_{d}^{f_{s}} \subseteq C L_{d}$.

With the above definitions, we address the RMFCSA design problem. Specifically, the aim is to find the candidate lightpaths which are to be assigned with an objective to reduce the (i) FSs amount that is utilized in the network's any MCF's any core, and (ii) aggregate FSs amount which is allocated within the network. The aforementioned is required to be achieved under the constraints of (i) assignment of demand successfully i.e., for each demand $d \in D$, a permissible candidate lightpath must be assigned from within $C L_{d}$, and (ii) capacity of the multiple core fiber i.e., in any MCF $e \in \varepsilon$, the provided FS $f s \in F S$ can be utilized at most $\varepsilon$ times. For a given demand, the formulated ILP model allocates a candidate lightpath hence making a decision on its route, MF and SA. Further, a decision on the core in every MCF which occurs on the candidate lightpath's route is conducted on the basis of the FSs which are occupied. To perform the aforementioned, the formulated ILP model uses the following binary variables:

- $\quad z_{d, l}$, where $z_{d, l}=I$ when a demand $d \in D$ uses a candidate lightpath $c l \in C L_{d}$, else $z_{d, l}=0$.

- $x_{e, f s}$, where $x_{e, f s}=I$ when FS $f s \in F S$ is utilized in any core of the MCF $e \in \varepsilon$, else $x_{e, f s}=0$.

- $y_{f s}$, where $y_{f s}=I$ when FS $f_{s} \in F S$ is utilized in any core within any MCF, else $y_{f s}=0$.

With the aforementioned definitions and binary variables, aim of the formulated ILP model is to

$$
\text { Minimize } K=\sum_{f s \in F S} y_{f s}+a \sum_{d \in D} \sum_{c l \in C L_{d}} h o p_{c l} \cdot \delta_{c l} \cdot z_{d, l}
$$


The first term of the optimization objective function in (2) ensures the least $|F S|$ which is required hence reducing the FSs amount utilized in any MCF's any core in the network. The aforementioned objective however is a common metric of evaluation in regard to the SDM-bEON since within the network, FSs that are assigned in only a single core of an individual MCF may differ to the FSs amount which are assigned in the aggregate cores of all the MCFs. Keeping in view of the aforementioned, second term of the objective function in (2) targets the reduction of the aggregate FSs amount which are allocated in the network. In the second term of (2), $\alpha$ is a very small positive number which is real valued, $h o p_{c l}$ the candidate lightpath $c l \in C L_{d}$ hop(s) number(s), and $\delta_{c l}$ the CFSs amount which is required by candidate lightpath for the communication process. Hence, the formulated ILP model ensures that the chosen $|F S|$ value is the one which results in the allotment of least FSs aggregate number even though multiple solutions may result in a similar $|F S|$ value.

The optimization objective function in (2) is constrained by the following:

$$
\begin{aligned}
& \sum_{c l \in C L_{d}} z_{d, l}=1, \quad \forall d \in D \\
& \sum_{d \in D_{c l \in C} \sum_{d}^{e} \cap c l_{d}^{f_{s}}} \sum_{z_{d, l}} \leq|\varepsilon| \cdot x_{e, f_{s}}, \forall e \in E, f_{s} \in F S \\
& \sum_{e \in E} x_{e, f_{s}} \leq|E| \cdot y_{f_{s}}, \forall f s \in F S
\end{aligned}
$$

Constraint (3) ascertains, for each demand in the set of demands, allocation of a candidate lightpath which is unique. Constraint (4) ensures that at maximum, $f_{s}$ FSs are employed by $\varepsilon$ lightpaths at the MCF $e$. Constraint (5) guarantees the appropriate value assignment to $y_{f s}$ under the account for such FSs which are utilized.

The following two points must also be highlighted in regard to our current study: (i) we have not considered grooming of the traffic which implies that any F-TP is assigned to at most one demand and further, every demand needs exactly a single lightpath assignment, and (ii) for any specific MCF link, we have not considered the allotment of a specific core owing to the assumption of the core switching flexibility provisioned by the F-OXCs nodes. Specifically, in the current work, instead of allocating the candidate lightpaths to particular $\operatorname{MCF}(\mathrm{s})$ core(s), based on their spectral resources which are available, the candidate lightpaths are assigned in any MCF core along the route.

\section{B. Heuristic Algorithm}

Number Existing studies in literature have already shown that ILP modelling for the design of EONs incurs $N P$-hard complexity and hence, it is ineffective when large sized networks are considered [1,2]. Therefore, for the design of SDM-b-EONs, which is an extension of the EONs design, requiring solution to RMFCSA problem, the ILP model is certainly not an effective strategy.

In view of the aforementioned, in this sub-section we design a heuristic algorithm based on the Simulated Annealing (SA) strategy that solves a similar problem as detailed in the previous sub-section however, generating practical solutions in reasonable amounts of execution times. The proposed heuristic algorithm, named as SArelying-RMFCSA (SA-r-RMFCSA), is designed by modifying the Simulated Annealing Greedy Lightpath Allocation (SAGLA) heuristic algorithm from our previous study [1] such that the SA-r-RMFCSA algorithm is able to find those candidate lightpaths that provision all the demands in the set $D$ under the consideration of the graph $G$ with an aim to satisfy the objective function given by (2). Further, the SA-rRMFCSA algorithm uses a simple greedy-RMFCSA (gRMFCSA) process to find an initial solution by provisioning fast solutions to the various demand(s) order(s) instants of the RMFCSA problem.

In regard to the SA method, it is known that the SA process generally admits, with a certain probability, solutions which are of the non-improving type [12, 13]. The starting temperature coefficient $(T)$ is useful in allowing the SA process to admit such non-improving type solutions with a certain probability which increase the value of the thus far found final solution's objective function. Also, it must be noted that, even if admitted, a non-improving solution may not be the optimum solution; however, it may permit the SA process to escape a local optimum value. Lastly, with the evolution of the heuristic algorithm, the probability of non-improving solutions' acceptance minimizes since; $T$ is reduced by a specific cooling rate value in every iteration.

In SA-r-RMFCSA, the SA procedure (i) aims to find a viable solution space so as to locally evaluate the solutions, and (ii) guides the g-RMFCSA process which obtains fast solutions by processing the demands one after the other as per a specific demand order followed by the assignment of lightpaths to the demands so as to reduce the objective function given by (2). The output of the SA-r-RMFCSA algorithm is a solution that provides the chosen candidate lightpaths set and the maximum iteration numbers. Further, the following must be noted in regard to the SA-r-RMFCSA algorithm: (i) in the gRMFCSA procedure, the shortest candidate lightpaths 


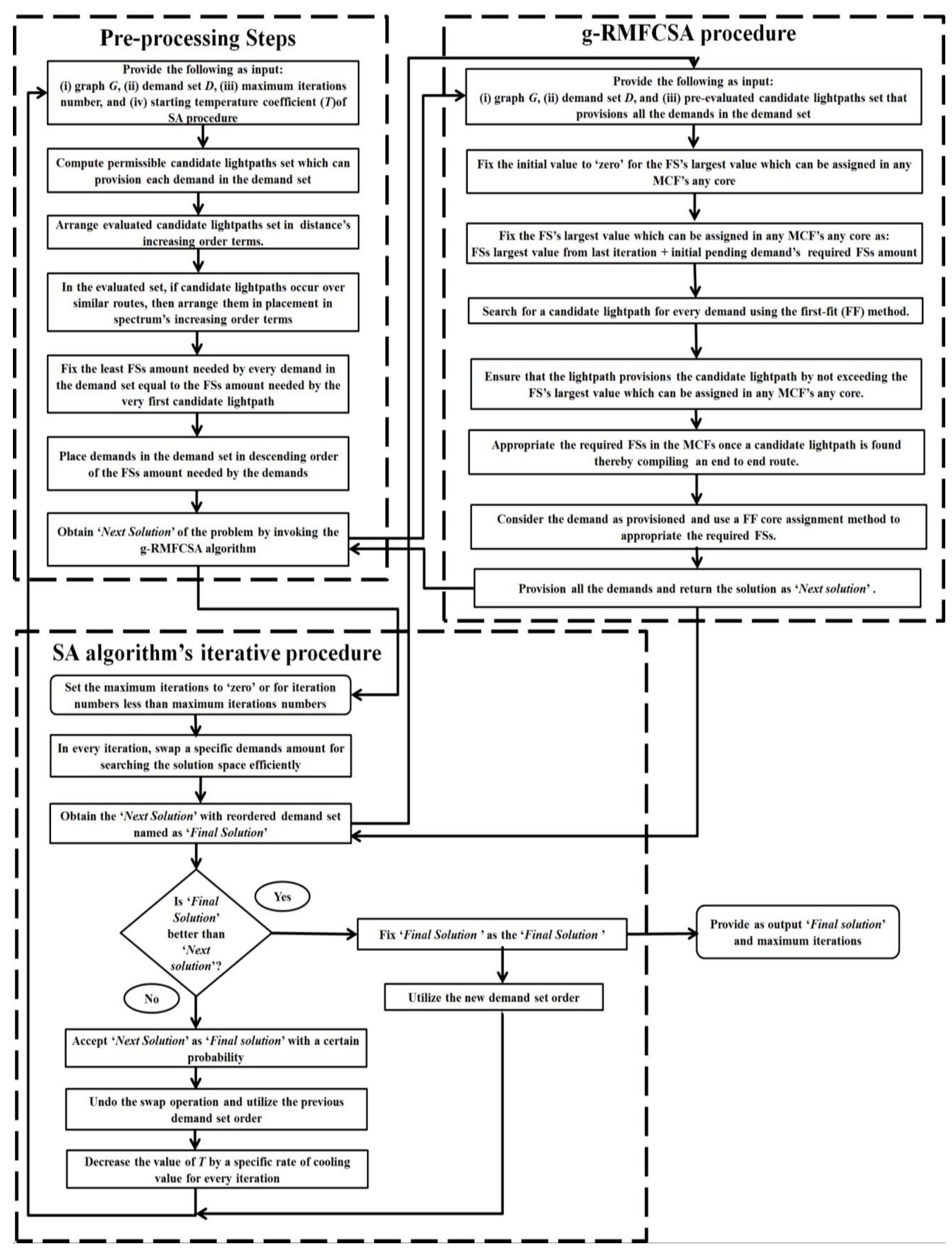

Fig.2. Flowchart of the SA-r-RMFCSA algorithm.

which have the least spectral parts are tried initially since, the candidate lightpaths in the set are increasingly arranged in terms of both, physical distance and spectral positioning, and (ii) in the SA algorithm's iterative procedure, before obtaining the 'Next Solution' from the g-RMFCSA algorithm, we perform a swap of the 
demands. The aforementioned in conducted in view of the fact that a MCF enabled SDM-b-EON can provide large capacity and also may require the provisioning of large numbers of demands. Thus, if only an individual demands pair is swapped, it may result in solutions that only marginally vary from each other, in turn leading to the SA process not being able to move away from the local optimum value. Therefore, we swap two large demands sub-sets which are ordered. Flow-chart of the SA-r-RMFCSA algorithm is as shown in Fig. 2.

\section{SiMULATION RESULTS}

For performance evaluation, we consider three realistic network topologies: the Deutsche Telekom (DT), the Telefónica (TID), and the GEANT. The details of these topologies with their various dimensions values can be found in our previous study [1]. In the aforementioned considered networks, we assume that (i) at start, in every core, there is an availability of complete $4 \mathrm{THz}$ of the $\mathrm{C}$ Band, (ii) the spectrum which is available is split into 12 $\mathrm{GHz}$ FSs [14] hence resulting in 320 FSs in every core, and (iii) the F-TPs deployed at the network nodes can operate at either 40/100/400 Gbps, and can use any of the following MFs: BPSK, QPSK, 16-QAM or 64-QAM. Following the study in [7], we conduct the simulations considering 'seven', 'twelve' and 'nineteen' cores MCFs and further, adopt the most limiting TR values assuming that either nonlinear interference (NLI) or ICXT dominates as the prominent degradation. The TR values are used by the proposed SDM-b-EON design strategy (i.e., ILP model or SA-r-RMFCSA) which always resorts to use of the most efficient MF based on these TR values. Lastly, a $10 \mathrm{GHz}$ spectral GB value is also considered between contiguous connections [15].

As for the demands set, we load the considered networks with demands set consisting of unidirectional demands and following an optimum distribution [16]. Further, we consider two profiles of traffic (PoT): (i) multiple-rate PoT (MR-PoT) which considers connections to take the values which correspond to 40/100/400 Gbps, and (ii) flexible PoT (F-PoT) which considers size of connection to range from 'one' to the 'maximum FSs' value. To consider realistic scenarios, we assume that MR-PoT is a less period case wherein, the $40 / 100 / 400$ Gbps demands occur as $35 \%, 55 \%$, and $10 \%$ of the total demands which are offered whereas, F-PoT is a long period case wherein, only 100 and 400 Gbps demands occur as $45 \%$ and $55 \%$ of the total offered demands. A similar study can also be conducted considering alternative traffic models in view of investigating PoT's impact on SDM-b-EON performance.

It must also be noted that the $400 \mathrm{Gbps}$ demands have very low TR and this leads to such demands not reaching the destination irrespective of the MF which is adopted. In the current study, in the case when, even though after resorting to the use of a less efficient MF, a $400 \mathrm{Gbps}$ demand is found to be impermissible during the candidate lightpath pre-evaluation process, we construct its corresponding candidate lightpath considering 'four' 100
Gbps lightpaths which follow both, contiguous assignment and joint switching between source and destination. The aforementioned however incurs four times the FSs amount which is required in addition to 40 $\mathrm{GHz}$ of GBs. The current study can however be extended by considering a translucent SDM-b-EON scenario in which the advanced MFs can be utilized in longer lightpaths which will be the focus of our future work.

A. Tuning of SA-r-RMFCSA Algorithm and Choice of Number of Shortest Paths

The performance of the SA-r-RMFCSA algorithm is dependent on the SA process which in turn requires tuning of the following parameters: (i) rate of cooling for each iteration, (ii) starting temperature coefficient $(T)$, and (iii) maximum iteration numbers. Therefore, before proceeding with the performance evaluations, we tuned the aforementioned SA parameters. We conducted extensive simulations in regard to the optimization objective function in (2) considering various demand values with both PoTs for the considered network topologies with 'nineteen' core MCFs accounting for the variations of amount of FSs allocated, FSs amount, and iteration numbers. From the obtained results, we report those values which demonstrated the 'best' performance (see Table 1), and for the remaining simulation experiments we use the same values. In regard to the maximum iteration numbers, it is known that, increase in the SA iterations value does not necessarily result in an improvement of objective function rather, it only leads to increased times of executions. Hence, in our simulations we fix the maximum iterations number to 10000 .

Table 1. The SA-r-RMFCSA tuning procedure results.

\begin{tabular}{cccc}
\hline $\begin{array}{c}\text { Network } \\
\text { Topology }\end{array}$ & $\begin{array}{c}\text { Rate of } \\
\text { Cooling for } \\
\text { each iteration }\end{array}$ & $\begin{array}{c}\text { Starting } \\
\text { Temperature } \\
\text { Coefficient }(T)\end{array}$ & $\begin{array}{c}\text { Maximum } \\
\text { Iteration } \\
\text { Numbers }\end{array}$ \\
\hline DT & 0.999 & 0.65 & 10,000 \\
TID & 0.998 & 0.58 & 9000 \\
GEANT & 0.998 & 0.54 & 9500 \\
\hline
\end{tabular}

To generate $C L_{d}$ for each $d \in D$, we resort to the use of $k$-shortest paths ( $k$-SPs) algorithm. However, to decide on the most appropriate value of ' $k$ ' to be used in the subsequent experiments, initially, we conducted simulation experiments considering the maximum numbers of cores or fibers (i.e., 'nineteen') in the GEANT topology which also presents worst results (see sub-section 3.3). The obtained results are shown in Fig. 3.

From Fig. 3(a) it can be observed that in order to minimize the $|F S|$, between the source and the destination, rather than traversing only the one $(k=1) \mathrm{SP}$, the candidate lightpaths must be permitted to span substitute routes. This occurs owing to the fact that when the candidate lightpath is constrained to only the one SP, compared to the border portion, where the FSs remain underused, link congestion in the network's middle portion is much higher resulting in the requirement of large FSs amount. However, this $|F S|$ minimization 
lowers as the $k$ value increases and further, for $k \geq 5$ and above, this reduction ends. The aforementioned occurs since (i) larger hop(s) amount(s) is spanned by the longer routes which in turn requisites that, to provision lightpath (s) over such routes, larger FSs are assigned, and (ii) traversing of longer distances implies TR issues leading to the usage of lesser efficient MFs.

Contrary to the behaviour of $|F S|$ observed in Fig. 3(a), from Fig. 3(b) it be seen that as the value of $k$ increases, there also occurs an increase in the aggregate FSs allocated since, there occurs an availability of longer routes with larger $k$ values. Also, the aforementioned effect occurs more in the MF cases since, the long TR permits the SA-r-RMFCSA algorithm to use long routes since the primary aim of the algorithm is a reduction of $|F S|$; however, this simultaneously results in an increase in the aggregate FSs allocated within the network. Overall, following the obtained results in Fig. 3, we set the value of $k=5$ in our simulations also noting that larger values will only result in increased times of execution for the SA-RMFCSA algorithm.

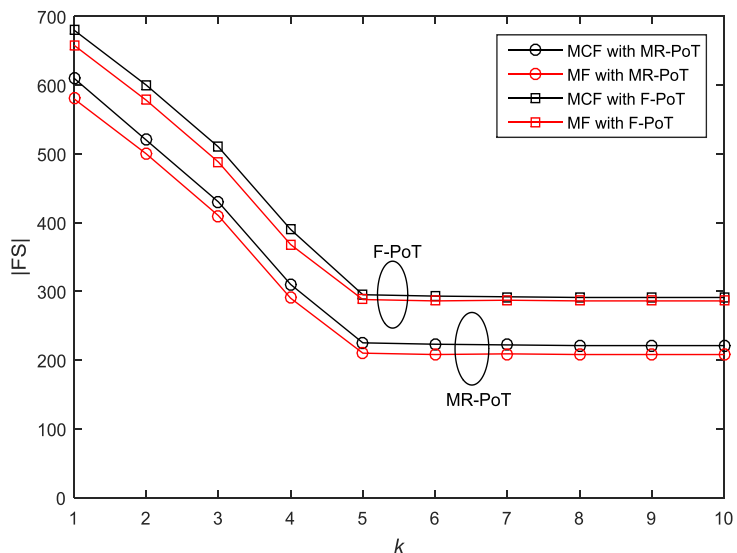

(a)

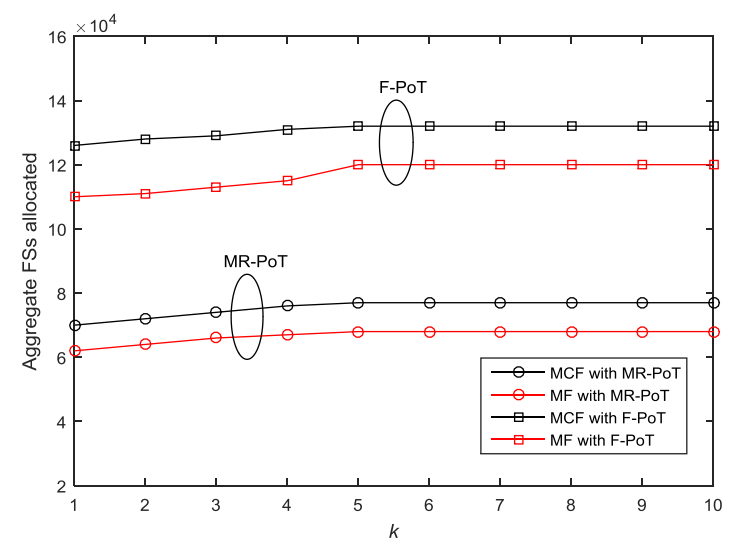

(b)

Fig.3. For a MCF and MF enabled SDM-b-EON consisting of 'nineteen' cores or fibers in the GEANT topology, variation of $k$ with (a) FSs amount, and (b) aggregate FSs allocated.

\section{B. Performance Comparison of ILP Model and SA-r- RMFCSA Algorithm}

In this sub-section, we compare performance of the
ILP model with that of the SA-r-RMFCSA algorithm considering the DT network. To find solutions of the ILP model, we use the CPLEX optimization software [17]. For this set of the simulations we assume $|D| \in\{200,400,600,800,1000\}$ as the randomly generated demands all of which follow MR-PoT. Also, for every run of the ILP model, the CPLEX solver is set to execute for a maximum time of 24 hours. We show the obtained results in Table 2 in the terms of $|F S|$, the aggregate FSs amount allocated, the execution times, $\Lambda_{F S}$ and $\Lambda_{\text {aggregate FSs }}$, which indicate the performance gaps in terms of FSs and total assigned FSs.

It can be observed from Table 2 that irrespective of the demand size, both, ILP model and SA-r-RMFCSA are able to obtain approximately similar $|F S|$. When the demand size is 800 and 1000, a difference of 2 and $3|F S|$ is observed between the ILP model and SA-rRMFCSA, respectively to be required in any MCF's any core of the DT network. The aforementioned results in $\Lambda_{F S}$ values of $2.98 \%$ and $2.77 \%$, respectively. In regard to the aggregate FSs amount allocated, it can be observed that SA-r-RMFCSA is able to obtain close performances to that shown by the ILP model with a maximum $\Lambda_{\text {aggregate FSs }}$ value of $3.41 \%$ for all the executions. As for the execution times, with larger demand values, the ILP model requires more than 24 hours to obtain the solutions whereas, the maximum execution time required by the SA-r-RMFCSA heuristic is of 285 seconds which demonstrates that the ILP model indeed has a limit on its scalability.

\section{Performance Comparison of SDM-b-EON with $M C F$ versus MF Technology}

Having established the suitability of the SA-rRMFCSA algorithm in the previous sub-section, in this sub-section we use it to compare the performance of a MCF and MF enabled SDM-b-EON. It must be noted that the major difference in the use of MCF and MF technology lies in the fact that, in the MF technology the amplified spontaneous emission (ASE) noise is a major factor that leads to limitation of the TR [3] whereas, in the MCF technology the TR is limited substantially due to ICXT which results in an inefficient use of resources and also renders the advanced MFs impermissible [7]. For this set of simulations we assume that a MCF enabled SDM-b-EON has 'seven', 'twelve' or 'nineteen' cores whereas a MF enabled SDM-b-EON has the same numbers of fibers in every link. We consider the TID and GEANT networks which are loaded with 5000, 10000, and 15000 demands under F-PoT or MR-PoT. For both the networks, in Fig. 4 we show the obtained results in regard to the FSs amount and the aggregate allocated FSs.

It can be observed from Fig. 4 (a) and (c) that when GEANT network is considered, for the 'seven' and 'twelve' cores or fibers case, in terms of both, FSs amount and aggregate FSs allocated, there occurs approximately no difference between the results obtained 
by $\mathrm{MCF}$ and MF technologies. The aforementioned observation confirms that the usage of relatively large values of cores do not necessarily result in SDM-b-EON performance degradation. The aforementioned occurs specifically because when the cores amount is (i) 'seven', TR is limited only by the ASE noise i.e., ICXT does not affect the performance, and (ii) 'twelve', only the 40 Gbps MFs are affected by ICXT which is counteracted by the large TR of the QPSK MF. From the results, it can also be observed that the aforementioned does not result in additional usage of the resources since the presence of
ICXT results in only few 40 Gbps lightpaths in the MCF scenario to adopt the QPSK MF compared to their analogous lightpaths in the MF scenario which opt for the 16-QAM or the 64-QAM MF; however, all the aforementioned MFs requisite the same amount ('two' because of $10 \mathrm{GHz}$ GB assumed in the study) of FSs which enable similar resource(s) usage.

With increase in cores amount to 'nineteen', IXCT starts to affect both, the $40 \mathrm{Gbps}$ and $100 \mathrm{Gbps}$ lightpaths which in turn has a detrimental effect on obtained results.

Table 2. Performance comparison of the ILP Model and the SA-r-RMFCSA algorithm.

\begin{tabular}{|c|c|c|c|c|c|c|}
\hline & \multicolumn{3}{|c|}{ ILP Model } & \multicolumn{3}{|c|}{ SA-r-RMFCSA } \\
\hline$|D|$ & $F S$ & $\begin{array}{l}\text { Aggregate FSs } \\
\text { allocated }\end{array}$ & $\begin{array}{l}\text { Execution times } \\
\text { (sec.) }\end{array}$ & $F S \mid\left(\Lambda_{F S} \%\right)$ & $\begin{array}{c}\text { Aggregate FSs allocated } \\
\left(\Lambda_{\text {aggregate } F S s} \%\right)\end{array}$ & $\begin{array}{c}\text { Execution } \\
\text { times (sec.) }\end{array}$ \\
\hline 200 & 21 & 1185 & 11600 & $21(0)$ & $1189(0.33)$ & 3 \\
\hline 400 & 39 & 2670 & 26500 & $39(0)$ & $2685(0.56)$ & 15 \\
\hline 600 & 48 & 3580 & 76111 & $48(0)$ & $3670(2.51)$ & 36 \\
\hline 800 & 67 & 4860 & $>24$ hours & $69(2.98)$ & 4940 (1.64) & 80 \\
\hline 1000 & 108 & 7620 & $>24$ hours & $111(2.77)$ & $7880(3.41)$ & 285 \\
\hline
\end{tabular}

Specifically, between the results obtained for the MCF and MF technology, it results in a performance difference of $12-13 \%$ and $11-12 \%$ in terms of FSs amount and aggregate FSs allocated, respectively. However, the aforementioned performance gaps also occur due to the assumption of a $400 \mathrm{Gbps}$ lightpath being provisioned as 'four' 100 Gbps lightpaths.

From Fig. 4 (b) and (d) similar aforementioned result trends in terms of both, FSs amount and aggregate FSs allocated can be observed for the TID network. However, compared to the GEANT network, the links in the TID network are much shorter which results in the TID SDMb-EON enabled by MCF to obtain much closer results to its MF counterpart as compared to the GEANT SDM-bEON. Specifically, in the case of 'nineteen' cores or fibers, the difference in obtained results remains lesser than $6.67 \%$ and $9.09 \%$ for the FSs amount and aggregate FSs allocated, respectively.

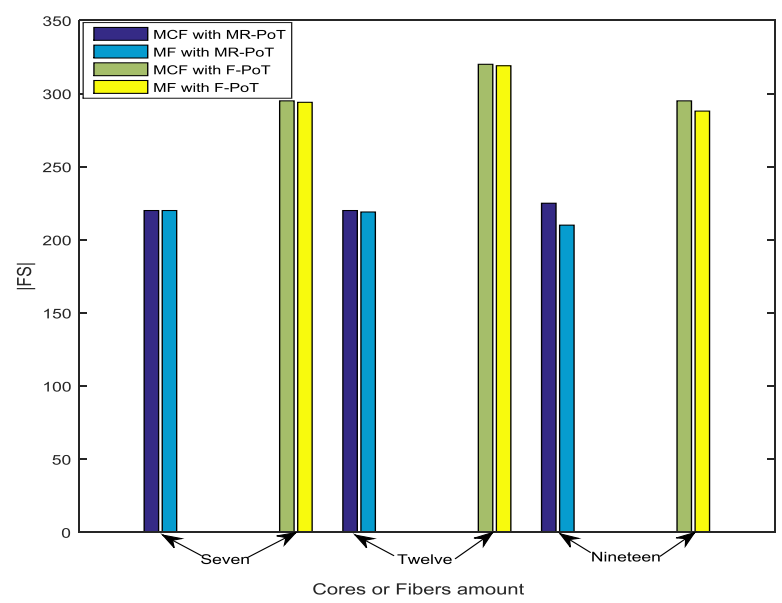

(a)

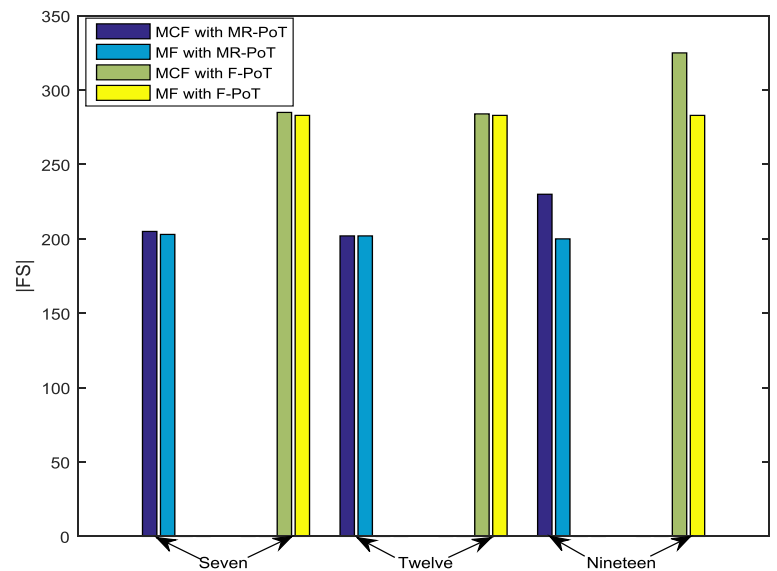

Cores or Fibers amount

(b)

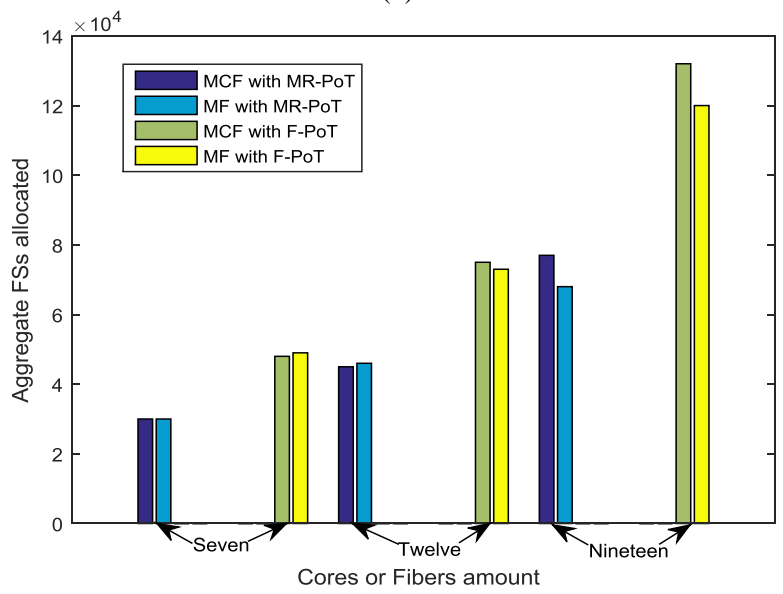

(c) 


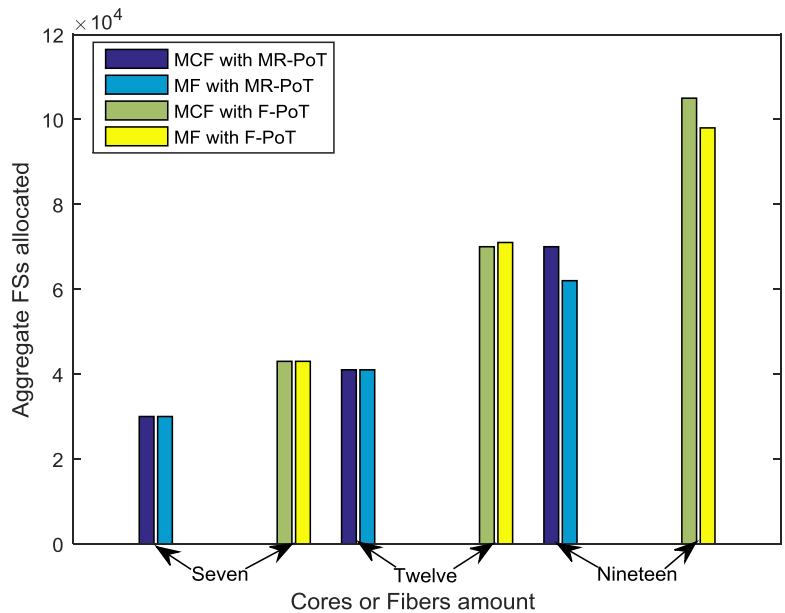

(d)

Fig.4. For a MCF and MF enabled SDM-b-EON consisting of 'seven', 'twelve' and 'nineteen' cores or fibers (a) FSs amount utilized in GEANT, (b) FSs amount used in in TID, (c) aggregate FSs allocated in GEANT, and (d) aggregate FSs allocated in TID.

After having established that the performance differences in MCF and MF enabled SDM-b-EON occurs only at larger values (i.e., 'nineteen') of the core, we now aim to investigate the reason for the occurrence of such differences. Specifically, we focus on the values of BR(s) and usage of $\mathrm{MF}(\mathrm{s})$ by the F-TPs which are under operation in a 'nineteen' cores or fibers SDM-b-EON case. The obtained results are shown in Fig. 5.

It can be observed from Fig. 5 (a) that when GEANT network is considered with the 'nineteen' cores MCF, use of the advanced MFs is either very limited (e.g. 16-QAM) or does not occur at all (e.g. 64-QAM). The aforementioned occurs owing to the fact that in the GEANT network, between the source and the destination, the lightpaths are required to traverse longer distances in addition to the presence of ICXT over the MCFs which enhances the limit on TR. On the other hand, it can be observed that irrespective of the BR, almost all the F-TPs which are operational resort to the use of QPSK MF. From Fig. 5 (c) it can be observed that when GEANT network is considered with the 'nineteen' fibers per MF link, there occurs more usage of advanced MFs since in this scenario, ASE noise is the major TR limiting factor. Specifically, in both, MR-PoT and F-PoT cases, F-TPs operating at 100 Gbps majorly use 16-QAM and 64QAM MFs. It can also be observed that no F-TP resorts to the use of BPSK MF since, compared to the QPSK MF, irrespective of the BR, it provisions no benefit in the TR. Finally, with the results shown in Fig. 5 (a) and (c), it can be inferred that the assignment of lightpaths in the network can be conducted efficiently provided advanced MFs are utilized. Through the aforementioned we are able to justify the marked differences in results between the SDM-b-EON using 'nineteen' MCFs/MFs technology that were observed in Fig. 4.
From Fig. 5 (b) it can be observed that that when the TID network is considered with the 'nineteen' cores MCF, compared to the case when the GEANT network was considered, majority of the F-TPs are able to utilize advanced MFs such as 16-QAM and 64-QAM owing to the TID's shorter link distances. Due to the aforementioned, as observed in Fig. 4, the efficiency in resource(s) usage is approximately equal to that obtained by the 'nineteen' fibers/link MF case. Further, during the simulations we also found that in the TID network with MCFs case, owing to impermissible TR, no 400 Gbps request requisited its provisioning as 'four' $100 \mathrm{Gbps}$ lightpaths; however, the aforementioned occurred multiple times in the GEANT network with MCFs case which resulted in larger numbers of F-TPs being required in case of every demand. Further, when the MF case was considered, the aforementioned effect was even more prominent since, at various instants, the SA-r-RMFCSA heuristic resorted to the use of longer 'four' $100 \mathrm{Gbps}$ lightpaths rather than utilizing a single 400 Gbps lightpath in view of minimizing the network FSs amount however needing more numbers of F-TPs. The aforementioned effect can be reduced by incorporating the minimization of F-TPs as an aim within the optimization function which will be the topic of interest in our future study.

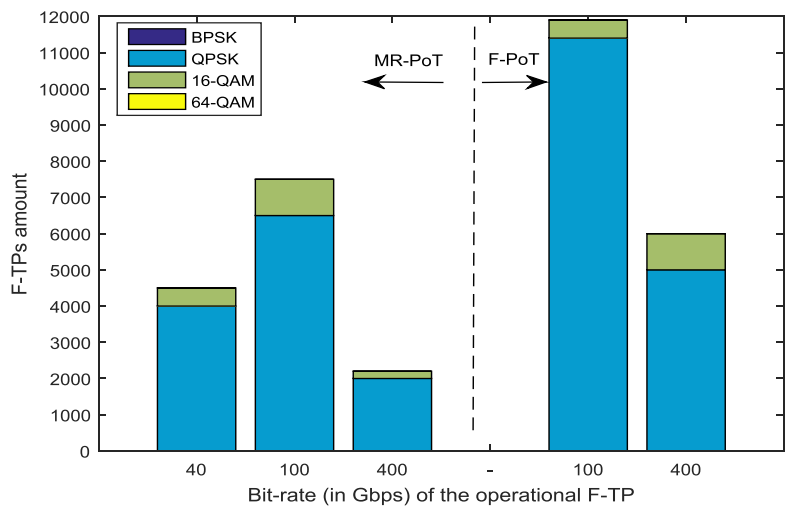

(a)

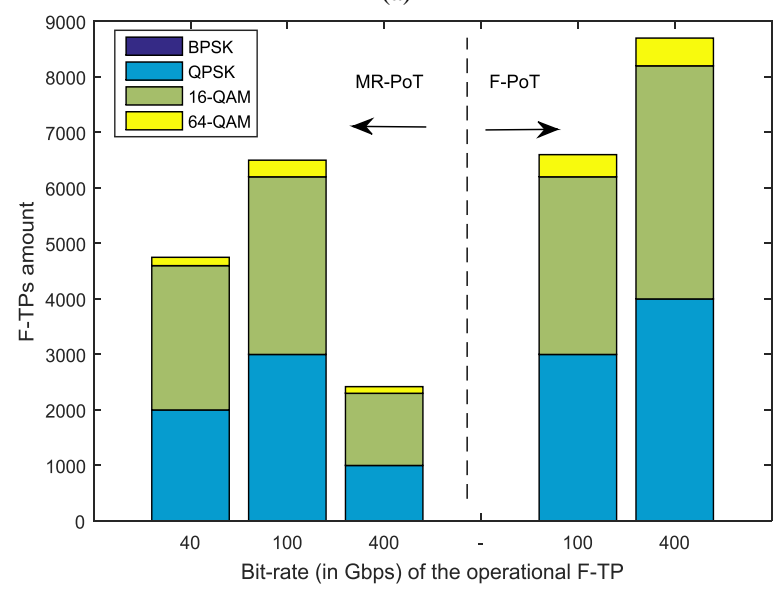

(b) 


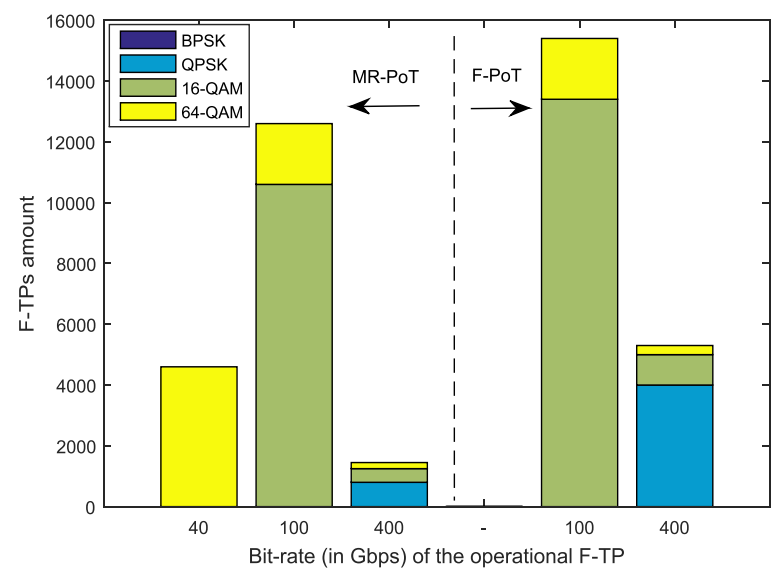

(c)

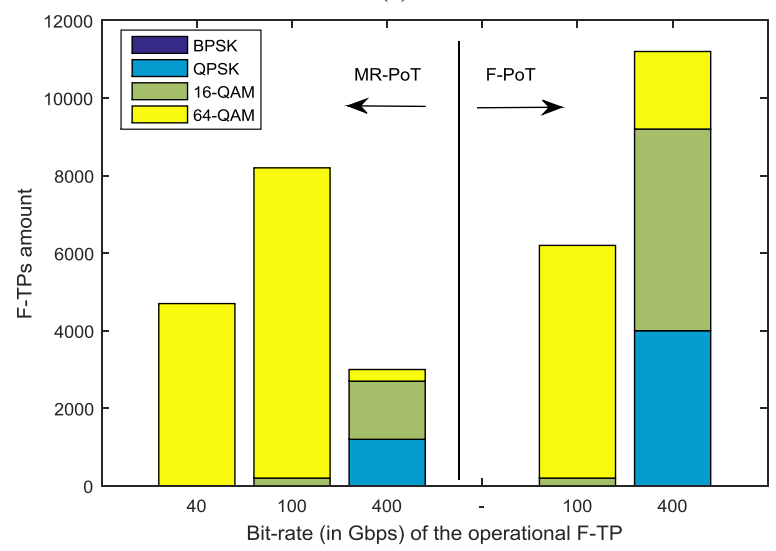

(d)

Fig.5. For a SDM-b-EON, following MT-PoT and F-PoT, the numbers of F-TPs operating at 40/100/400 Gbps in (a) GEANT with 'nineteen' core MCFs, (b) TID with 'nineteen' cores MCFs, (c) GEANT with 'nineteen' fibers per MF link, and (d) TID with 'nineteen' fibers per link.

\section{CONCLUSION}

In the current work, we have compared the performance of a MCF and MF enabled SDM-b-EON. Initially, we designed the SDM-b-EON using an ILP formulation followed by the proposal of the SA-rRMFCSA heuristic algorithm in view of obtaining solutions for large sized networks in reasonable execution times. For simulations, we considered realistic parameters and network topologies which are characterized by different node(s) numbers and link distances. The obtained performance comparison results demonstrated that the performance of the MCF enabled SDM-b-EON is very close to that of the MF enabled SDM-b-EON. Hence, our study establishes that benefits can be obtained from the use of existing components for MCFs rather than incurring any extra expense to provision the same traffic amount, and the aforementioned information is helpful to the network operators.

\section{REFERENCES}

[1] S. Iyer, "Traffic Grooming with Survivability and PowerEfficiency in Software Defined Elastic Optical Networks", J. of Opt., Springer, vol. 47, pp. 351-365, 2018.

[2] B. Chatterjee, N. Sarma, E. Oki, Routing and spectrum allocation in elastic optical networks: A tutorial, IEEE Commun. Surv. \& Tutorials, vol. 17, pp. 1776-1800, 2015.

[3] P. S. Khodashenas, J. Manuel Rivas-Moscoso, D. Siracusa, F. Pederzolli, B. Shariati, D. Klonidis, E. Salvadori, I. Tomkos, Comparison of Spectral and Spatial Superchannel Allocation Schemes for SDM Networks, IEEE J. Lightwave Technol., vol. 34, pp. 2710-2716, 2017.

[4] P.J. Winzer, Optical networking beyond WDM, IEEE Photonics J., vol. 4, pp. 647-651, 2012.

[5] D. Klonidis, F. Cugini, O. Gerstel, M. Jinno, V. Lopez, E. Palkopoulou, M. Sekiya, D. Siracusa, G. Thouénon, C. Betouleet, Spectrally and Spatially Flexible Optical Network Planning and Operations, IEEE Commun. Mag., vol. 53, pp. 69-78, 2015.

[6] P.J. Winzer, Spatial Multiplexing in Fiber Optics: The 10x Scaling of Metro/Core Capacities, Bell Labs Tech. Journal, vol. 19, pp. 22-30, 2014.

[7] F.J. Moreno-Muro, R. Rumipamba-Zambrano, P. PavónMarino, J. Perelló, J.M. Gené, S. Spadaro, Evaluation of Core-Continuity-Constrained ROADMs for FlexGrid/MCF Optical Networks, IEEE/OSA J. of Opt. Commun. Netw., vol. 9, pp. 1041-1050, 2017.

[8] P.J. Winzer, Making spatial multiplexing a reality, Nature Photon., vol. 8, pp. 345-348, 2014.

[9] N. Amaya, M. Irfan, G. Zervas, R. Nejabati, D. Simeonidou, J. Sakaguchi, W.Klaus, B.J. Puttnam, T. Miyazawa, Y. Awaji, N. Wada, I. Henning, Fully-elastic multi-granular network with space/frequency/time switching using multi-core fibres and programmable optical nodes, Opt. Express, vol. 21, pp. 8865-8872, 2013.

[10] A. Muhammad, G. Zervas, D. Simeonidou, R. Forchheimer, Routing, Spectrum and Core Allocation in Flexgrid SDM Networks with Multi-core Fibers, in Proceedings of Optical Network Design and Modeling (ONDM), pp. 192-197, 2014.

[11] A. Muhammad, G. Zervas, R. Forchheimer, Resource Allocation for Space-Division Multiplexing: Optical White Box Versus Optical Black Box Networking, IEEE/OSA J. Lightwave Technol., vol. 33, pp.4928-4941, 2015.

[12] M. Gendreau, J.Y. Potvin, Handbook of Metaheuristics, second ed., Springer U.S.A, 2010.

[13] M.S.G. Tsuzuki, Simulated Annealing - Single and Multiple Objective Problems, In Tech Open, 2012

[14] ITU-T recommendation G.694.1, Spectral grids for WDM applications: DWDM frequency grid, 2012.

[15] O. Gerstel, M. Jinno, A. Lord, S.J. Ben Yoo, Elastic Optical Networking: A New Dawn for the Optical Layer?, IEEE Commun. Mag., vol. 50, pp. s12-s20, 2012.

[16] F. Liu, J. Comellas, Optimized traffic profile for FlexGrid optical networks, Photon. Netw. Commun., Springer, vol. 34, pp 334-343, 2017.

[17] IBM. ILOG CPLEX optimizer. (2017). Available: http://www.ibm.com. 


\section{Authors' Profiles}

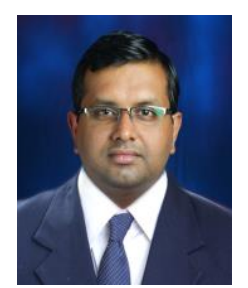

Sridhar Iyer received the B.E. degree in Electronics and Telecommunications Engineering from Mumbai University, India in 2005, M.S degree in Electrical and Communication Engineering from New Mexico State University, U.S.A in 2008, and the Ph.D. degree from Delhi University, India in 2017. He worked as an Assistant Professor in the Department of ECE at NIIT University, and Christ University, India between 2012-2016. He received the young scientist award from the Department of Science and Technology, Government of India in the year 2013. Currently he is an Associate Professor in the Department of ECE, Jain College of Engineering, India. His research interests include the architectural, algorithmic, and performance aspects of the optical networks, with current emphasis on efficient design and resource optimization in Space Division Multiplexing enabled flexi-grid Elastic optical networks. Dr. Iyer has published over 60 peer-reviewed articles in the aforementioned areas.

How to cite this paper: Sridhar Iyer, "Multiple Core Fibers versus Multiple Fibers Enabled Space Division Multiplexing Based Elastic Optical Networks: A Performance Comparison", International Journal of Computer Network and Information Security(IJCNIS), Vol.11, No.8, pp.13-23, 2019.DOI: 10.5815/ijcnis.2019.08.02 